\title{
Semantic Building Information Model and Multimedia for Facility Management
}

\author{
Christophe Nicolle and Christophe Cruz \\ LE2I UMR CNRS 5158, University of Bourgogne, BP 47870, 21078 Dijon Cedex, France \\ \{cnicolle, christophe.cruz\}@u-bourgogne.fr
}

\begin{abstract}
In the field of civil engineering, the proliferation of stakeholders and the heterogeneity of modeling tools detract from the quality of the design process, construction and building maintenance. In this paper, we present a Web-based platform lets geographically dispersed project participants-from facility managers and architects to electricians to plumbers-directly use and exchange project documents in a centralized virtual environment using a simple Web browser. A 3D visualization lets participants move around in the building being designed and obtain information about the objects that compose it. This approach is based both on a semantic architecture called CDMF and IFC $2 \times 3$. Our framework, based on Building Information Modeling features, facilitates data maintenance (data migration, model evolution) during the building lifecycle and reduces the volume of data.
\end{abstract}

Keywords: Interoperability, 3D collaborative platform, Industry foundation classes (IFC), Building information modeling (BIM), Semantic web.

\section{Introduction}

For twenty years, the construction sector makes a profound challenge. This is due to several causes such as: evolution of standards, evolution of methodologies and pressure on controlling costs and delays. The building lifecycle management requires the development of a specific environment solving at the same time the problems of syntactic and semantic heterogeneity $[14,15]$. Moreover, the environment should also allow the required extensibility and the flexibility in order to guarantee the coherent evolution of the collaborative processes developed in this field. The information in an AEC project is generated during all the building lifecycle. It is essential to structure the information in a relevant way for a better management. The activities in an AEC project generate a huge variety of data and information. Consequently, the management and the communication of these data by various participants are complex. The process of information sharing requires a framework in which computer programs can exchange data automatically regardless of the software and data location. Moreover, in this field, the use of tools for 3D visualization of the buildings is crucial. Towards this goal the IAI proposed a standard called IFC that specifies object representations for AEC projects [5]. Industry foundation classes (IFCs) include object specifications, or classes, and provide a structure for data sharing among applications.

J. Filipe and J. Cordeiro (Eds.): WEBIST 2010, LNBIP 75, pp. 14-29, 2011.

(C) Springer-Verlag Berlin Heidelberg 2011 
From a collaborative point of view the IFCs form the basis of a building description. This basis is enriched during the building's lifecycle with elements related to facilities management: financial data, maintenance rules, evacuation procedures and so on. The quantity of information becomes exponential and then a relevant organization of these elements becomes very complex. Today, "Building Information Modeling (BIM)" is promising to be the facilitator of integration, interoperability and collaboration in the future of building industry. The term BIM has been recently pointed to demarcate the next generation of Information Technologies (IT) and Computer-Aided Design (CAD) for buildings.

The remainder of this paper is structured in 3 parts. The first part presents a brief description of the concept of BIM. The second part presents the principles of our approach using semantic graphs to describe the objects of the building. The third part presents the industrial platform derived from our work and examples to illustrate our point.

\section{Building Information Modeling}

The term BIM marks the transition from conventional CAD applications for construction and recent developments in computer science. It is the process of generating, storing, managing, exchanging and sharing building information in an interoperable and reusable way. A BIM system is a tool that enables users to integrate and reuse the information of a building and the domain knowledge throughout the lifecycle of a building [16]. A BIM system is a central system that manages various types of information, such as enterprise resource planning, resource analysis packages, technical reports, meeting reports, etc. However, the main feature of a BIM is the 3D modeling system with data management, data sharing and data exchange during the lifecycle of the building. As a matter of fact, a building is composed of geometrical elements which are the basis of a building's design. Furthermore, parametric modeling provides powerful mechanisms that can automate the generation of the building information. Especially those mechanisms, in conjunction with the behavior of building object and an object-based system, facilitate the maintenance and the validity of the building's designs. Several definitions of BIM can be found in the specialized literature. The NBIMS [17] divides BIM categories in three axes which are Product, Collaborative Process and Facility. The Product is an intelligent digital representation of the building. The Collaborative Process covers business drivers, automated process capabilities and open information standards used for information sustainability and fidelity. The Facility concerns the well understood information exchanges, workflows, and procedures which are used by the different teams as a repeatable, verifiable and sustainable information-based environment throughout the building's lifecycle. According to [18], a BIM is a computable representation of all the physical and functional characteristics of a building and it is related to the project information, which is intended to be a repository of information for the building owner/operator to use and maintain throughout the lifecycle of the building. According to Autodesk [19], BIMs have three main features: They create and operate on digital databases for collaboration. They manage change through those databases 
so that a change to any part of the database is coordinated in all other parts. They capture and preserve information for reuse by adding industry-specific applications.

By analyzing the BIM definition we index a set of features common to BIM systems $[20,21,22,23,24,25,27]$. (1) The main feature of BIM is the ability to store, share and exchange data. Many methods are used to realize those processes like files or databases. Concerning data exchange, BIMs are developed with the aim to keep open non-proprietary data format exchange. (2) Data managed in BIM processes concerns building geometries which are most of the time 3D data. 3D data is more helpful for designers for the visualization of complex construction conditions than 2D while it communicates at the same time design intentions. AEC industry visualizes the design using stereoscopic projection tools to create an immersive experience [26]. Spatial relationships between building elements are managed in a hierarchical manner. (3) BIMS are data rich and comprehensive as they cover all physical and functional characteristics of a building. BIMs are also rich semantically as they store a high amount of semantic information about building elements. Moreover, the data model is fully object oriented to facilitate data management and process definition. (4) Some of the BIMs are extensible to cover unimplemented information domains. For instance, the development of IFC 2.X went though a major change in order to extend progressively the range and the capability of IFCs by using modules. (5) BIMs play a central role in the building lifecycle. In order to ease data exchange, a data format has to be widely used. By definition, BIMs enable interoperability among diverse applications using a shared universal information standard. (6) The lifecycle of the project in AEC is composed of several phases which have to be validated by the corresponding AEC engineering designer. BIMs cover several lifecycle phases. The state of these phases is processed by BIMs in order to sequence and schedule the process. BIMs support $4 \mathrm{D}$ analysis, where activities from the project schedule can be simulated and studied to optimize the sequence of construction.

Our research aims at solving the problem linked to the constant IFC evolution (4). The definition of a complete framework that allows to manage knowledge around the building process requires an extensible and generic formalism to represent both specific data describing building information and connected information defined by the user during the building's lifecycle. It requires also tools to handle and query the corresponding modeled data, and it requires also tools to manage the data evolution during the building's lifecycle. Moreover, the contextual management of data that needs to correspond with the user's view and constraints has to be taken into account. It requires also an adaptive graphical 2D/3D representation, dynamically connected with data from buildings according to the BIM features. Finally, the most important point is the fact that the framework has to take into account the constant evolution of specific data describing building information and the corresponding connected information defined by the user during the building's lifecycle. We have developed a method that combines IFC and the various requirements related to facility management.

\section{Overview of Our Approach}

In our study context the requirement of the model extensibility and the model evolution generates others difficulties, such as mapping data between two models. Handling information during the building lifecycle requires a contextual and temporal 
representation of knowledge. It is important to trace each data evolution at a time and to know how to present data according to the user context $[6,7]$. To deal with these requirements, we derived Names Graph [4] in order to complete our framework. Based on the context we developed a system description and operators in an architecture called CDMF that allows dealing with the traceability of the data schema evolution. This innovative approach allows knowing, at any time of the project, the current version of the data schema that defines the facility data.

Our approach considers all requirements at large (temporal management, adaptive view, 2D/3D representation) in order to propose a global solution with a framework based on Semantic Web technologies. To meet these requirements, we have built a complete framework, called CDMF, derived from Semantic Web formalism: RDF, Named Graph, OWL and SWRL. These formalisms constitute the base of our approach. We have extracted from each of them the more adapted features to our problematic. RDF formalism allows data modeling and can be used by operators provided by OWL/SWRL. Finally, Named Graph gives a contextual layer to this unit. To obtain a complete formalism, well adapted to facilities management, we have defined a framework called CDMF which will be presented in the following section.

\subsection{The CDMF Architecture}

The CDMF architecture proposes to use semantic operators in order to manage data in the context of a facility management environment. The objective of CDMF is to join together the semantics of OWL and SWRL in only one formalism. For that DMF defines a whole system of logical operators allowing the description of classes, properties, constraints and of rules. The principal interest of CDMF is to offer a framework facilitating the description of contextual data. This framework offers a single structure that permit us to define a set of data, all types of contexts and the actions that can be realized on these data. CDMF aims at meeting the various needs evoked; moreover, it achieves for the complete system, due to its structure and its operators, a reduction in volume of the data that represents an information system in a collaborative environment, as well as restricted treatments due to the unicity of information. Thus, we used the formalisms of the semantic Web to create an environment meeting in a single way our various needs.

The operators of DMF allow the modeling of knowledge on 3 levels (Table 1): the model level, where DMF makes it possible to define the concepts of modeling (class, property, etc). The diagram level, where DMF allows defining the description of knowledge. The instance level, which makes it possible to define the objects of the real-world according to the structure of the diagram defined in the higher level of abstraction. For each level a set of triplets forms RDF graphs.

Table 1. The 3 levels of the modeling data of DMF

\begin{tabular}{|c|c|c|}
\hline Model & Schema & Instance \\
\hline $\begin{array}{l}d m f: C l a s s \text { type dmf:Class } \\
\text { dmf:Property } \\
\text { rdf:Property }\end{array}$ & $\begin{array}{l}\text { :Building type dmf:Class } \\
\text { : Storey type dmf:Class } \\
\text { :contains } \\
\text { dmf:Property }\end{array}$ & $\begin{array}{l}\text { :b1 type : Building } \\
: \text { e1 type :Floor } \\
: \text { e2 type :Floor } \\
: \text { b1 :contain :s1 }\end{array}$ \\
\hline & & :b1 : contain :s2 \\
\hline
\end{tabular}


The architecture of CDMF is based on the structure of modeling RDF. This structure RDF makes it possible to represent knowledge with graphs. These graphs are modeled using a set of triplets. A triplet is composed of a subject, a predicate, and an object. The architecture of CDMF is composed of two layers: "DMF" and "C". The "DMF" layer is composed of the model construction operators and the " $C$ " layer is composed of the context manager operator and the handling graph operator.

The CDMF architecture is made of the space system stack, the API and the engine. This environment allows the creation of specific applications that permit to deal with facility management requirements. The Space System is used to configure the system and to allow data access. It is based on an RDF document. This space system contains a set of graphs called SystemGraph. From this point, the CDMF engine checks the declared graphs and responds to queries executed from the API. The API CDMF is a set of methods used to handle the data system. This API proposes to access data with two main classes called SystemSetGraph and SystemGraph. The first one allows to access the system graph which composes the space system. The second one is made of methods that permit to modify system data. For instance, the method SystemGraph.create() provides the list of Class elements and Property elements which can be created. The CDMF engine is the kernel of the architecture. This engine uses a space system to configure and to know the set of systems to use. The engine contains processes which manage methods of the API CDMF. The engine selects the system graphs in the space system, and creates data, deletes data, etc.

\subsection{DMF: A Reduced Set of Modeling Operators}

This section presents the DMF stack. This stack is made of operators which allow to model information (from simple and monovalued attributes to complex 3D objects) into semantic graphs. This section enables us to show that the formalism that we have defined has a restricted set of operators. We show that these operators can be combined to meet all the needs for semantic modeling defined in the statements. For each operator we give its equivalent in SWRL or OWL.

- dmf : Class defines a class. The equivalent operator in OWL is owl : Class.

- dmf : Property carries out the definition of a property of a Class.

- dmf : Equal defines the equality between two variables. This operator makes it possible to test if two resources are equivalent.

- "dmf : Var" makes it possible to define variables used in the logical formulas. Its equivalent is defined in SWRL by the operator "swrl : Variable".

- "dmf: Pred1" makes it possible to define unary predicates. Its equivalent is defined in SWRL by "swrl:ClassAtom".

- "dmf:Pred2" makes it possible to define binary predicates. The equivalent operators in SWRL are "swrl:IndividualPropertyAtom" and "swrl : DatavaluedPropertyAtom". A binary predicate is a property with a subject and an object. To make the correspondence with RDF, the terms of subject and object are used in order to define the first and last element of a triplet RDF. 


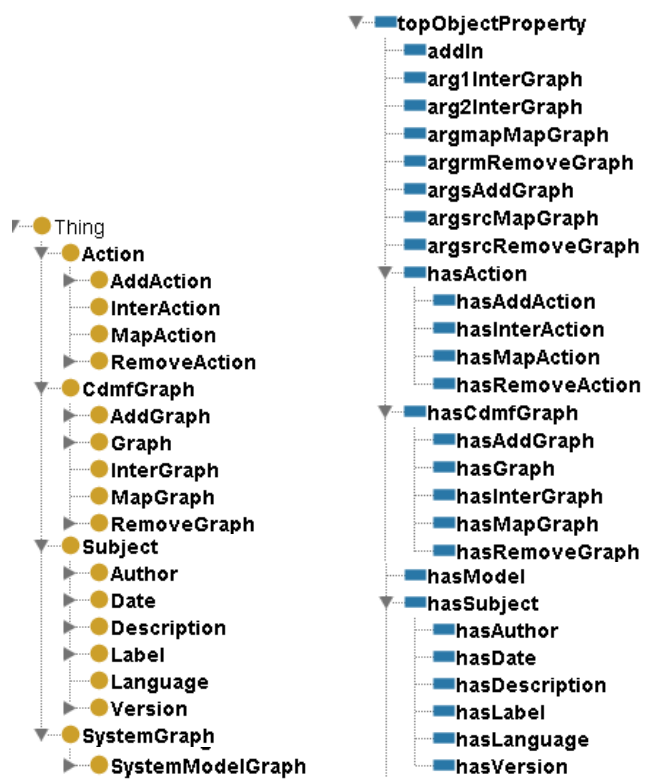

Fig. 1. Hierarchy of CDMF operators and properties using Protégé

- "dmf : Equiv" makes it possible to define that two classes are equivalent. The set of the elements of the type $\mathrm{A}$ is equivalent to the set of the elements of the type $\mathrm{B}$. The equivalent operator in OWL is "Owl : equivalentClass".

- etc.

\subsection{Context and Mapping Operators}

This section presents the operators defined in the stack $\mathrm{C}$ of our architecture. These operators are used to handle graphs and to define contexts. With these operators, new graphs can be generated by combination of existing graphs. These operators are commonly used to update the data model definition when a norm in architecture is upgraded. For example, the IFC norm has been updated six times since 2000. The elements defined in this part use the space of name cdmf. For each type of graph we present its definition by using DMF operators, as well as an example of use with the result of the operation carried out on the graphs.

Union Operator. The result of the addition of G1 and G2 is the union of the set of the triplets of G1 and the set of the triplets of G2. Table 2 gives its definition in DMF (Table 2).

Table 2. DMF definition of the union operator

\begin{tabular}{|c|}
\hline 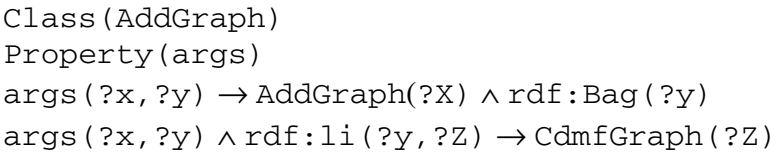 \\
\hline
\end{tabular}


The operator of a union of graphs is defined by the class cdmf : AddGraph. It has a property cdmf : args. This property is a list of RDF elements ( $r d f: B a g$ ) whose elements are graphs. The definition of these elements allows the union of two or several graphs. Table 3 shows an example of a union of graphs as well as the result of the operation.

Table 3. Script defining the union of two graphs G1 and G2

\begin{tabular}{|c|c|c|}
\hline :Ga1 & $r d f: c d m f$ & type:AddGraph \\
\hline :Ga1 & cdmf:args :li1 & \\
\hline : li1 & rdf:Li :G1 & \\
\hline : 1 i1 & rdf:Li :G2 & \\
\hline$:$ G1 \{ & $\begin{array}{l}\text { :b1 raf:type } \\
\text { :e1 raf:type }\end{array}$ & $\begin{array}{l}\text { :Building } \\
\text { :Floor }\end{array}$ \\
\hline$: G 2\{$ & G1:b1 G1: conta & $\operatorname{ain}:$ :e1 \\
\hline
\end{tabular}

G1 defines two objects b1 and e1 of the Building type and Floor. G2 defines a relation contain between $\mathrm{b} 1$ and $e 1$. The resources $\mathrm{b} 1$ and $\mathrm{e} 1 \mathrm{in} \mathrm{G} 1$ and $\mathrm{G} 2$ are the same resources because their URI is identical. Indeed b1 and e1 are defined in G1, their URI is the concatenation of the URI of G1 with b1. In G2, we define the namespace $\mathrm{G} 1$ with the URI of $\mathrm{G} 1$, therefore $\mathrm{G1}: \mathrm{b} 1$ and $\mathrm{Gl}: \mathrm{el}$ in $\mathrm{G} 2$ is the same URI as: b1 and:e1 in G1. The result of the calculation of this combination is presented in Table 4.

Table 4. Script resulting from the union of G1 and G2

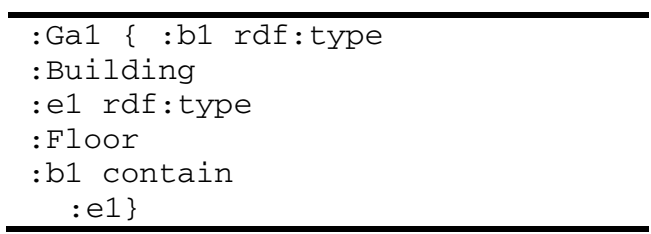

Intersection Operators. The intersection operator can be defined in different manners and can imply a different result according to the type of intersection carried out. The intersection operator is defined by two elements cdmf: InterGraph and cdmf : CompInterGraph. The first element defines a "traditional" intersection. The second element makes it possible to specify on which elements of a triplet the intersection is carried out.

Traditional Intersection. The result of the intersection between G1 and G2 is the set of the identical triplets in G1 and G2 (Table 5).

Table 5. DMF definition of the traditional intersection operator (cdmf:InterGraph operators)

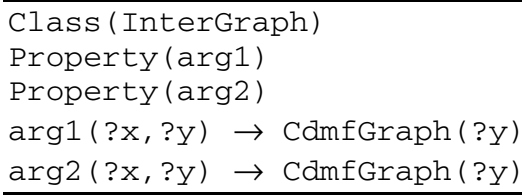


This operator has two properties cdmf:arg1 and cdmf:arg2. These two properties are CdmfGraph types representing the two graphs on which the intersection must be computed. Table 6 defines an intersection between the two graphs G1 and G2. The result of this intersection is the empty set, because the set of triplets is disjoint.

Table 6. Script defining the union of two graphs G1 and G2

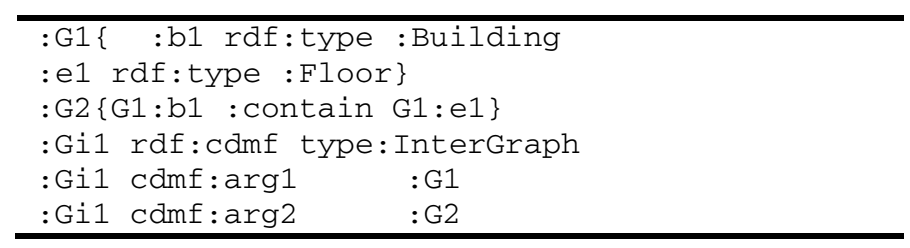

Composed Intersection. The composed intersection makes it possible to determine which part of the triplet is concerned in the calculation of the intersection. In the case of the "traditional" intersection, one carries out the intersection on the set of triplets of each graph (Table 7). Here we can compose the intersection with the various parts of a triplet (subject, object). Below you will find possible combinations of intersections.

- The intersection on the subject in the two graphs. The result of the intersection between $\mathrm{G} 1$ and $\mathrm{G} 2$ is the set of the triplets whose subjects are identical in G1 and G2.

- The intersection on the object in the two graphs. The result of the intersection between G1 and G2 is the set of the triplets whose objects are identical in G1 and G2.

- The intersection on the subject of the triplets of a graph with the object of the triplets of the other graph. The result of the intersection between G1 and G2 is the set of the triplets whose subjects of the graph G1 are identical to the objects of the graph $\mathrm{G} 2$.

- The intersection on the subject or the object. There is a last combination which is actually the addition of two intersections. The result of the intersection on the subject of G1 and the subject or the object of G2 is equivalent to the sum of the intersections on the subject of G1 and G2, and on the subject of G1 and the object of $\mathrm{G} 2$.

Table 7. DMF definition of the composed intersection operator (cdmf:CompInterGraph operators)

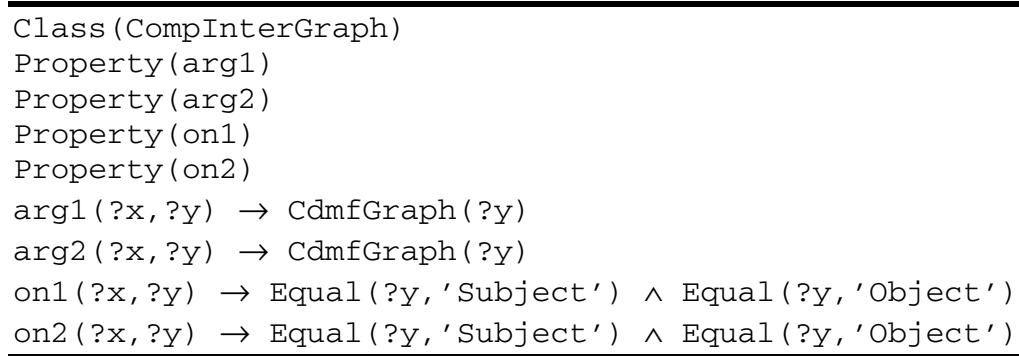


The intersection operator cdmf:CompInterGraph has two properties cdmf : $\arg 1$ and cdmf : arg2 which are the two graphs on which the intersection is carried out. It has two additional properties cdmf : on 1 and cdmf : on 2 respectively defining the two parts of the triplets used to carry out the calculation of an intersection. Table 8 defines a composed intersection between the graphs G1 and G2 previously defined (e.g. Table 3).

Table 8. Script defining the composed intersection of two graphs, G1 (on subject part) and G2 (on object part)

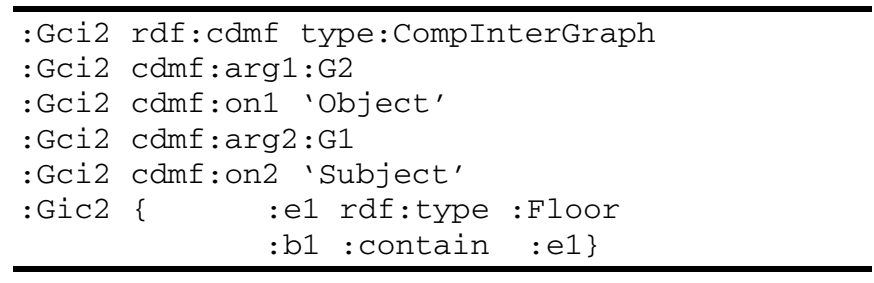

Difference Operator. The difference between two graphs is indicated by the element cdmf:RemoveGraph. The result of the difference between G1 and G2 is the suppression of the set of the triplets of G2 in G1 (Table 9).

Table 9. DMF definition of the difference operator

\begin{tabular}{ll}
\hline Class (RemoveGraph) & \\
Property (cdmf: src) & \\
Property (cdmf:rem) & \\
cdmf: src (?x, ?y) $\rightarrow$ & RemoveGraph(?x) \\
CdmfGraph (?y) & \\
cdmf:rem (?x, ?y) $\rightarrow$ & RemoveGraph(?x) \\
CdmfGraph (?y) & \\
\hline
\end{tabular}

The class cdmf : RemoveGraph has two properties cdmf : src and cdmf :rem. The second property constitutes the set of the triplets to be withdrawn from the graph indicated by the first argument.

Table 10. Script defining the difference between two graphs, G1 and G2.

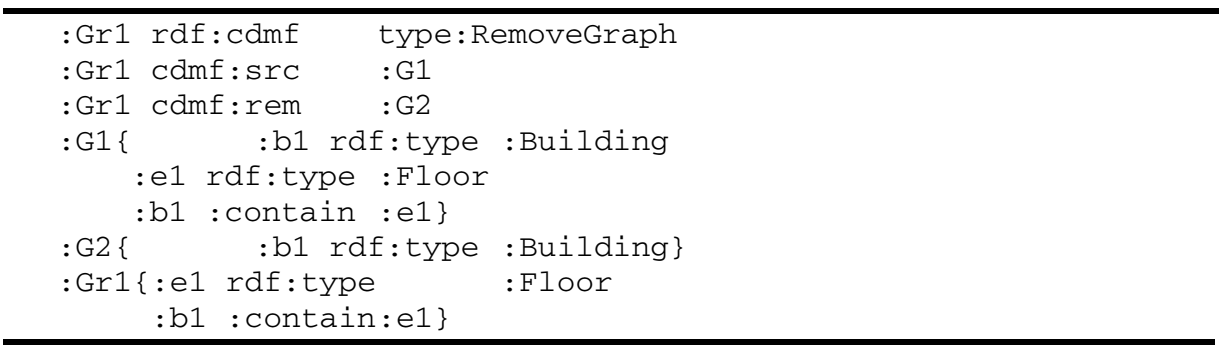

In this operation, only the identical triplets are a removed graph source. 
Mapping Operator. The last type of operation on the graphs is the operation of mapping described by the element cdmf:MapGraph. A graph of mapping is a transformation of a graph into another graph using mapping rules;

Table 11. DMF definition of the mapping operator

\begin{tabular}{|c|}
\hline $\begin{array}{l}\text { Class (MapGraph) } \\
\text { Property (src) } \\
\text { Property (map) } \\
\text { src (?x, ?y) } \rightarrow \text { MapGraph (?x)^ CdmfGraph (?y) }\end{array}$ \\
\hline $\operatorname{map}(? x, ? y) \rightarrow \operatorname{MapGraph}(? x) \wedge \operatorname{CdmfGraph}(? y)$ \\
\hline
\end{tabular}

The mapping operator has two properties $\mathrm{cdmf}: \mathrm{src}$ and $\mathrm{cdmf}:$ map indicating the source graph and the target graph. The result of the operation of mapping is the set of the triplets which is defined by the rules of Gmap. A rule in Gmap is described by an operator of implication. All the triplets of GsrC are transformed into triplets defined by the rules (Table $12 \& 13$ ).

Table 12. Script defining the mapping of graph Gsrc using the rules defined in a graph

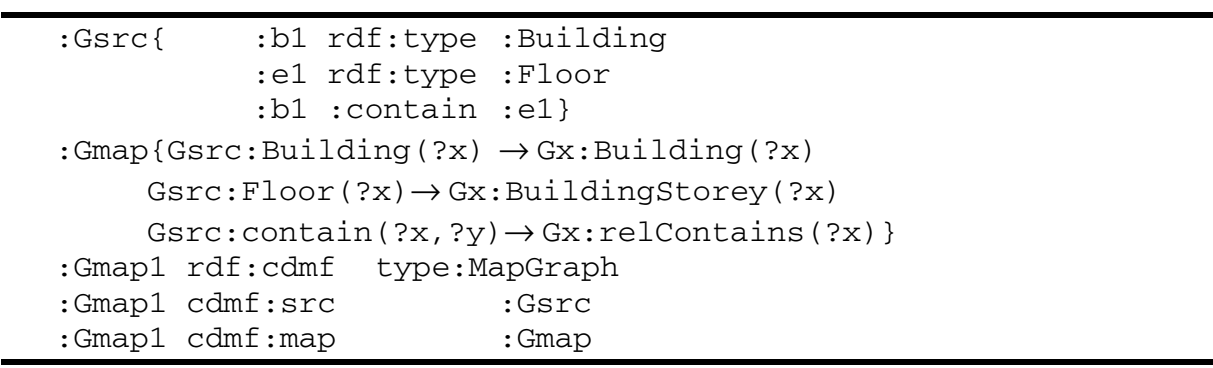

In this part we have studied five operators which allow to carry out various combinations of graphs. These five operators are the union, the difference, the intersection (traditional and composed) and the mapping. They constitute the first part of the $\mathrm{C}$ stack. The second part of the $\mathrm{C}$ operators is the definition of a particular graph SystemGraph. This element associates various types of information with a graph. This element is used to represent contexts.

Table 13. Script presenting the result of the mapping of the graph G1 previously defined (Table 3)

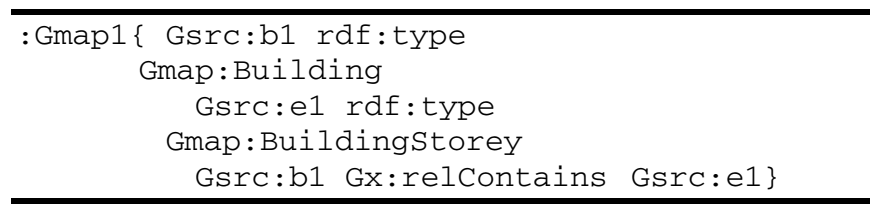




\subsection{Context Modeling, the Element SystemGraph}

The element cdmf : SystemGraph uses the mechanism of Named Graphs to define the contexts with the help of the properties. The SystemGraph element associates with the graphs presented above all useful information which is needed to respond to the set of problems met in facility management. This element defines the nature of the graph, on which a graph data model is based. The element also defines the context of use and actions that can be realized on this graph.

SystemGraph evokes the data model on which the associated graph is built. A protégé description of the SystemGraph is depicted in figure 2.

For instance, the definition of building $\mathrm{X}$ is based on a data model that describes the composition of a building. The data model allows to check the data coherence of the associated graph and allows to indicate which kind of data can be generated in the graph. This data model is defined with the help of operators introduced in DMF. In fact, the SystemGraph defines actions that can be undertaken on the graph such as reading, writing or deleting. This can be done according to the actions which are authorized on the associated graph. The description of the context in SystemGraph is a list of RDF resources. This section presents the definition of SystemGraph with its properties: cdmf:model, cdmf:of, cdmf:Action, cdmf: graph. The name space cdmf is used to present these elements.

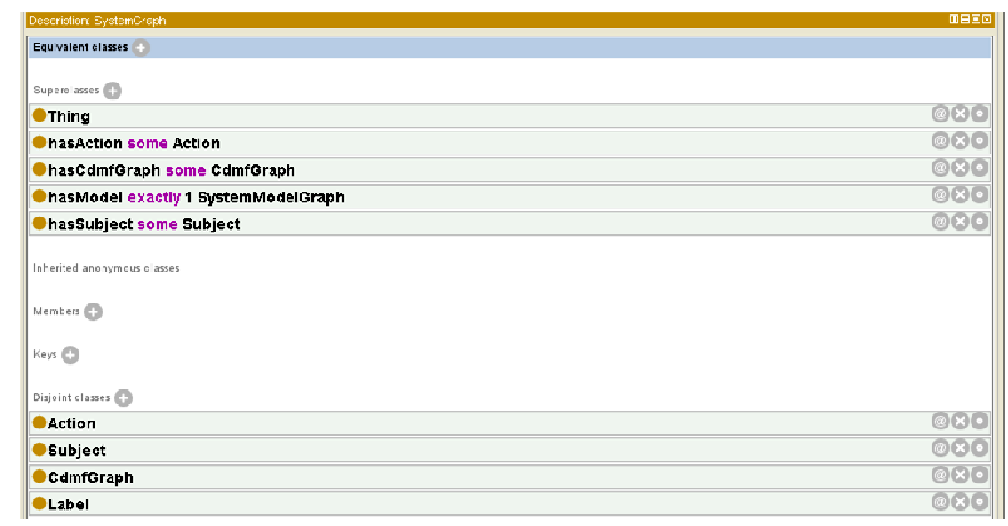

Fig. 2. Description of SystemGraph into Protégé

The property cdmf:model defines the model on which a SystemGraph element is based. The associated model will be used to define the objects and the properties which can be generated in the SystemGraph graph. Subsequently, it is possible to check the data coherency by comparing it to the model. SystemGraph has a model which is also a SystemGraph. CDMF defines a class cdmf: SystemModel Graph to represent a specific SystemGraph. This type of SystemGraph contains definitions of classes, properties and rules defined in the syntax DMF. 
The Property cdmf: of defines the subject of the SystemGraph element. This property defines the context. It associates a set of RDF resources which resume what is described by the SystemGraph. For instance, SystemGraph can be the description of a data model in the building field. SystemGraph can be a data model in a certain version or SystemGraph can represent data on a certain date and in a certain language, for a certain user. It can also define the nature of the graph and the conditions that have to be fulfilled in order to be able to access a graph system.

The property $\operatorname{cdmf}$ :Action determines the actions authorized on the graph. It defines the actions of writing, suppression and modification. If no action is associated to the system, this implies that only the visualization of information is possible.

The element cdmf : Action determines which actions are possible, on which part of the data and starting from which model.

An action has one or two properties. If it has only one property add then the addition is allowed. If it has only one property remove then the system allows the deletion of data. If it has the two properties, we can add and remove data in the graph system. An element cdmf:Add defines which information we can add (cdmf:model) and where it has to be added (cdmf: addIn).

The Element cdmf:Remove indicates the suppressible data which have to be removed. If it does not have this property, all the data of the graph of the system can be deleted. The property cdmf:From binds an element of the cdmf:From type. According to the origin of the suppression (cdmf: graph), this element defines the action to be realized: either an addition in a $c d m f:$ RemoveGraph, or a suppression in the graph cdmf: graph.

The property cdmf:graph contains the associated graph representing the data. The associated graph is a cdmf: graph type. SystemGraph has an attribute of the cdmf:graph type. Thus, SystemGraph can refer to all the types of graphs presented in CDMF.

\section{Active3D Facility Server}

This section presents the Active3D Facility Server, a web collaborative platform dedicated to the facility management, taking into account all aspects of the building's lifecycle. Due to the lack of space, we will illustrate only our proposal with two examples of use. The first example concerns the initialization of a space system when a facility manager needs to configure the platform. This extension is realized by defining a specific model. This model will be used in the building definition process. The second point illustrates the use of context to display specific information to users.

\subsection{Configuration of a SpaceSystem}

In facility management, various versions of the building can be managed and presented to different actors in many countries. The representation of a building mixes textual and graphic representations. The first step in facility management consists in creating the data model. A building description will be generated starting from this model. The new data model is created from a new applicative environment. An initial space system is created. The graphical representation is given in Fig. 2. 
Following this step, the facility manager can store his data in the graph and has the possibility to create data starting from SystemGraph 'Space System'. The creation of SystemGraph includes a model, a context (list of resources) and the graph of data. For the model, the facility manager has to choose among the SystemModelGraphs" available. A sytemModelGraph element is a SystemGraph whose characteristic it is to contain models in its associated graph. A SystemModelGraph represents only a DMF model. For the context, the list of resources contains only one resource where the representation is 'Building Model'.

For the associated graph, the facility manager creates a new RDF graph which will contain the definition of the model. The result is presented in Fig. 2.For AEC projects, we have based our model definition on the IFC 2X3. This model contains approximately 600 classes $[5,11]$. The following snapshot presents a part of the IFC model in the application. This model is created with the tools proposed by the application (creation of classes and properties).

Fig. 3 presents a snapshot of an IFC building. Each IFC object is represented in the Building Model by an operator dmf:Class. IFC links are represented by an operator dmf:Property. From this SystemGraph, we can declare classes, properties, rules, etc. In this example, we have created simply three classes which are Building, Floor and space and a property contain.

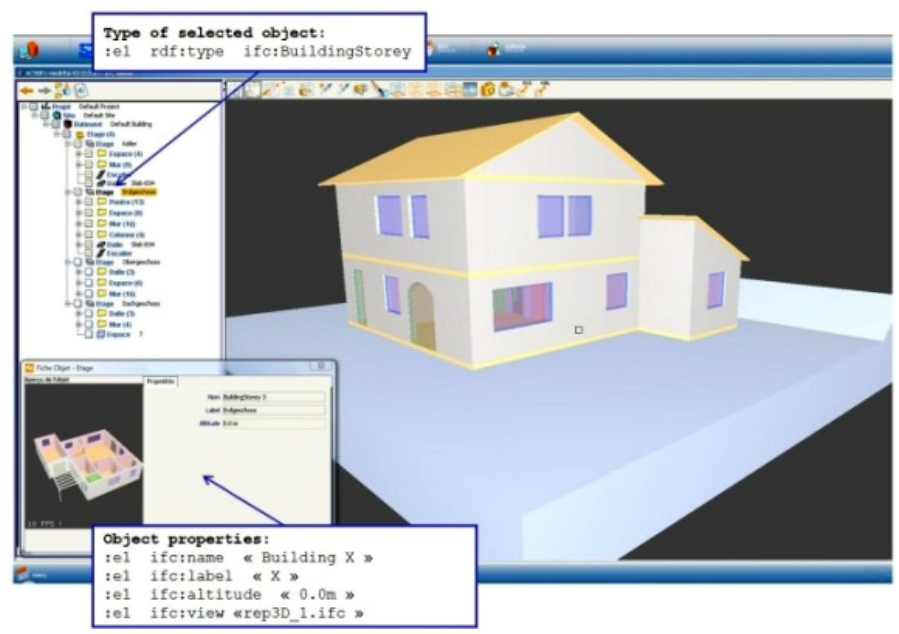

Fig. 3. An example of a 3D view of a building object in a Facility Management view using DMF operators

\subsection{Context Representation}

From the SystemGraph element, the facility manager defines a view on data according to a specific context. This context can be linked to a specific step in the building's lifecycle or it can be linked to a specific type of user (for example plumbers, architects or structure engineers). In the Active3D collaborative platform, this context is used to build user models that define data, operators and interface for a 


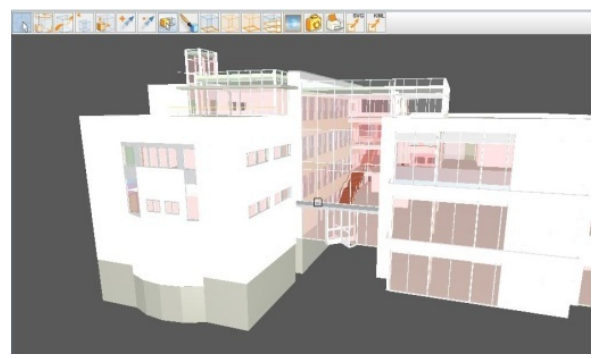

Fig. 4. Architectural view of a building. In this snapshot it is required to display the walls for the architect.

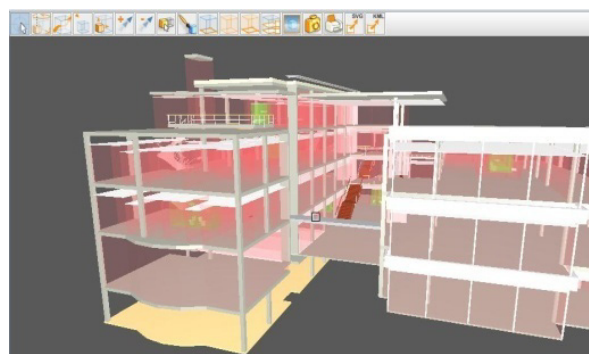

Fig. 5. Structural view of a building. This view is required for structure engineers. The corresponding graph provides all elements needed to make structure calculations.

specific user. Thus, during the identification protocol, when a user tries to connect himself to the platform, a specific graph is built and a view of a building is built according to its context. Figures 4 and 5 show two different views of the same building according to the Architect view and the Structure Engineering view respectively.

\section{Conclusions}

In this paper we have presented a Semantic Web approach for facility management. This approach allows facility managers to support the building's lifecycle management from the design to the destruction of the building in a collaborative context. Several actors provide and handle building information. This approach is based on a semantic model called CDMF and the IFC $2 \times 3$ standard which defines the 3D geometries of the objects of building. CDMF improves data management during the lifecycle of a building. Our proposition, based on graph combinations and the contextual element SystemGraph, addresses the problem of model evolution, of data mapping, of the management of temporal data, and of the adaptation of data according to the use and the user. Our framework facilitates data maintenance (data migration, model evolution) during the building lifecycle and reduces the volume of data. A collaborative Internet platform was developed to support the building's lifecycle. This platform is mainly used to federate all the actions realized on a building during its lifecycle, to merge all information related to these actions in an adaptive hypermedia graph, to extract some trade views of the building by combining information collected during the lifecycle from heterogeneous sources and to handle all these views through a dynamic and adaptive 3D interface. Currently, the Active3D platform supports more than 70 million square meters of building where more than 400 actors from all civil engineering domains collaborate at each step of the building's lifecycle. Semantic management of these millions of object requires some optimizations to reduce the data extraction time from semantic graphs. To meet these new challenges, we are working on a new semantic engine using based technique on the Model Checking. The objective will be the rapid extraction and verification of relevant semantic graphs in different contexts of use. 


\section{References}

1. Klyne, G., Carroll, J.J.: Resource description Framework (RDF): Concepts and Abstract Syntax (2004), http: / /www .w3 . org/TR/rdf-concepts /

2. McGuinness, D.L., Van Harmelen, F.: OWL Ontology Language: Overview (2004), http: / / www.w3.org/TR/owl-features/

3. Horrocks, I., Patel-Schneider, P.F., Boley, H., Tabet, S., Grosof, B., Dean, M.: SWRL: A Semantic Web rule Language: Combining OWL and RuleML (2004), http: / / www .w3 . org/Submission/SWRL/

4. Carroll, J.J., Bizer, C., Hayes, P., Stickler, P.: Named Graph. Provenance and Trust (2005), http: / /www2 005.org/cdrom/docs/p613.pdf

5. http://www.iai-international.org (2007)

6. Guha, R. V., Fikes, R.: Contexts for the semantic web. In: McIlraith, S.A., Plexousakis, D., van Harmelen, F. (eds.) ISWC 2004. LNCS, vol. 3298, pp. 32-46. Springer, Heidelberg (2004)

7. Guha, R.V.: Contexts: A Formalization and Some Applications. PhD thesis (1995)

8. McCarthy, P.: Introduction to Jena (2004), http://www-128.ibm.com/developerworks/java/library/j-jena/

9. Prud'hommeaux, E., Seaborne, A.: SPARQL Query Language for RDF (2005), http: / /www.w3 .org/TR/rdf-sparql-query/

10. Bizer, C., Cyganiak, R.: NG4J Named Graph API for Jena (2005), http://sites.wiwiss.fu-berlin.de/suhl/bizer/ng4j/

11. IFC 2x3 Workshop, Boston, USA (March 19, 2007), http://127.0.0.1:4664/redir?url=http $\% 3 \mathrm{~A} \% 2 \mathrm{~F} \% \mathrm{Fbuildingsmart} \% 2 \mathrm{E}$ Com\%2Eau2 F\&SrC=1\&schema=2\&s=suabHoX9yEfSmitJ zdEaD6NuYKQ

12. Cruz, I.F., Xiao, H., Hsu, F.: An Ontology-based Framework for Semantic Interoperability between XML Sources. In: Eighth International Database Engineering \& Applications Symposium (July 2004)

13. Klein, M.: Interpreting XML via an RDF schema. In: ECAI workshop on Semantic Authoring, Annotation \& Knowledge Markup, Lyon, France (2002)

14. Keith, A., Atkin, B., Bröchnet, J., Haugen, T.: Facilities Management, Innovation and Performance. Taylor and Francis Edition (2004) ISBN 0415321468

15. Barrett, P., Baldry, D.: Facilities Management, Towards Best Practice. Blackwell Publishing (2003) ISBN 0632064455

16. Lee, G., Sacks, R., Eastman Charles, M.: Specifying parametric building object behavior (BOB) for a building information modeling system, Automation in Construction. Knowledge Enabled Information System Applications in Construction, vol. 15(6), pp. 758-776. Elsevier, Amsterdam (2006)

17. NBIMS: National Building Information Modeling Stan-dard Part-1: Overview, Principles and Methodologies, US National Institute of Building Sciences Facilities Information Council, BIM Committee (2007),

http: //www. facilityinformationcouncil.org/bim/publications. php

18. NBIMS: National BIM Standard Purpose, US National Institute of Building Sciences Facilities Information Council, BIM Committee (2006),

http: / /www.nibs.org/BIM/NBIMS_Purpose.pdf

19. Autodesk White Paper: Building Information Modelling (2002), http://images.autodesk.com/apac_sapac_main/files/4525081_BIM _WP_Rev5.pdf 
20. Tolman, F.: Product modelling standards for the building and construction industry: past, present and future, Automation in Construction, 8th edn., pp. 227-235 (1999)

21. NIST CIS2 Web Site, Web Site (Available online at (2007), http://cic.nist.gov/vrml/cis2.html

22. Eastman, C., Wang, F., You, S.F., Yang, D.: Deployment of an AEC industry sector product modelo. Computer Aided Design 37(12), 1214-1228 (2005)

23. Zamanian, K.M., Pittman, J.H.: A software industry perspective on AEC information models for distributed collaboration, Automation in Construction, vol. 8(3), pp. 237-248 (1999)

24. SABLE Web Site, Web Site (Available online at (2005), http: / /www.blis-project.org/sable/

25. Cruz, C., Nicolle, C.: Active3D: Vector of Collaboration, Between Sharing and Data Exchange, INFOCOMP. Journal of Computer Science 5(3), 1-8 (2006)

26. Campbell., D.A.: Building Information Modeling: The Web3D Application for AEC. In: ACM Web3D, Perugia, Italy (2007)

27. Vanlande, R., Nicolle, C., Cruz, C.: IFC and Buildings Lifecycle Management. Journal of Automation in Construction (2008) 